\title{
Two domains of anosognosia in Alzheimer's disease
}

Sergio E Starkstein, Liliana Sabe, Erán Chemerinski, Laura Jason, Ramón Leiguarda

\begin{abstract}
Objective-To examine the presence of different dimensions of unawareness in patients with probable Alzheimer's disease.

Methods-A consecutive series of 170 patients with probable Alzheimer's disease were assessed with the anosognosia questionnaire-dementia (AQ-D) which includes items related to cognitive deficits and behavioural problems.
\end{abstract}

Results-A factor analysis of the AQ-D produced two factors: a "cognitive unawareness" factor, which loaded on items of memory, spatial and temporal orientation, calculation, abstract reasoning, and praxis, and a "behavioural unawareness" factor which loaded on items of irritability, selfishness, inappropriate emotional display, and instinctive disinhibition. A stepwise forward regression analysis showed significant correlations between the cognitive unawareness factor and more severe cognitive deficits, delusions, and apathy, but less depression. On the other hand, the behavioural unawareness factor correlated significantly with higher mania and pathological laughing scores. Whereas the cognitive unawareness factor showed a significant correlation with cognitive tests assessing verbal comprehension and long term memory, and was significantly associated with a longer duration of illness, no significant correlations were found between the behavioural unawareness factor and the neuropsychological tasks.

Conclusion-Unawareness of cognitive deficits and unawareness of behavioural problems may constitute independent phenomena in Alzheimer's disease. Whereas unawareness of cognitive deficits is related to the severity of intellectual impairment and the presence of delusional apathetic mood, unawareness of behavioural problems may be part of a disinhibition syndrome.

(F Neurol Neurosurg Psychiatry 1996;61:485-490)

Keywords: anosognosia; dementia; behaviour

Unawareness or the explicit denial of cognitive deficits has often been reported in patients with probable Alzheimer's disease. In a recent study that included a consecutive series of 103 patients with Alzheimer's disease we found that $20 \%$ had anosognosia, and a similar prevalence was reported by other authors. ${ }^{1-5}$ Most studies divided patients with Alzheimer's disease into groups with or without anosognosia based on the presence of unawareness for the cognitive (mainly memory) deficits. Whereas behavioural problems such as depression, irritability, emotional lability, disinhibition, and apathy are often found in Alzheimer's disease, whether patients with Alzheimer's disease may also show unawareness of behavioural problems has rarely been examined.

In a recent study, Vasterling et $a l^{6}$ reported that unawareness (measured as patient minus care giver disagreement) was most prominent in ratings of memory and self care, less prominent in ratings of anxiety and irritability, and absent in ratings of depression and health status. Whereas these findings suggest a dissociation between unawareness of cognitive deficits and unawareness of behavioural problems, Vasterling et al used an anosognosia instrument with unknown validity and reliability, cognitive and behavioural domains were arbitrarily defined, and the low depression ratings produced by patients with Alzheimer's disease may have produced a "floor" effect that explains the low correlation between depression and anosognosia scores.

The mechanism of anosognosia in Alzheimer's disease still remains unknown. Several studies showed that patients with Alzheimer's disease with anosognosia had significantly more severe deficits on "frontal lobe related" neuropsychological tests (such as set alternation tasks) compared with nonanosognosic patients with Alzheimer's disease, ${ }^{7-8}$ but other studies could not replicate those findings ${ }^{15}$ One possibility is that anosognosia for cognitive deficits and anosognosia for behavioural problems have different neuropsychological and psychiatric correlates. In this case, the association between anosognosia and deficits in specific cognitive tasks may be primarily related to the prevalence of different domains of anosognosia within the Alzheimer's disease sample.

The aim of this study was to determine the presence of different domains of anosognosia in Alzheimer's disease. For this purpose we examined a large sample of consecutive patients with Alzheimer's disease using the anosognosia questionnaire-dementia (AQ-D). The AQ-D is a quantified scale with established reliability and validity in Alzheimer's disease, which includes questions about different cognitive deficits and behavioural prob- 
lems. ${ }^{1}$ To determine specific correlates of anosognosic domains, patients were also assessed with a comprehensive neuropsychological battery and a structured psychiatric evaluation.

\section{Materials and methods \\ PATIENTS}

We examined a consecutive series of 186 patients who attended the neurology clinic of our institute due to progressive cognitive decline. The inclusion criteria were the following: (1) NINCDS-ADRDA criteria for probable Alzheimer's disease, ${ }^{9}(2)$ normal results on laboratory tests, (3) no history of closed head injuries with loss of consciousness, strokes, or other neurological disorders with CNS involvement, (4) no focal lesions on CT or MRI, and (5) a Hachinski ischaemic score $<4$.

\section{PSYCHIATRIC EXAMINATION}

After informed consent, patients were assessed by a psychiatrist with a comprehensive psychiatric evaluation, which provided measurements of different behavioural domains such as depression, anxiety, anosognosia, apathy, irritability, pathological affect, disinhibition, and delusions.

\section{Structured clinical interview for DSM-III-R (SCID) ${ }^{\prime \prime}$}

The SCID is a semistructured diagnostic interview for making the major axis I DSMIII- $\mathrm{R}^{12}$ diagnoses. The SCID was administered by a psychiatrist blind to the remaining clinical data, and the interview was carried out with the patient and at least one first degree relative. Based on the SCID responses, DSM-III-R axis I diagnoses were made.

\section{Hamilton depression scale (HAM-D) ${ }^{13}$}

The HAM-D is a 17 item interviewer rated scale that measures psychological and autonomic symptoms of depression.

Hamilton anxiety scale $(H A M-A)^{14}$

The HAM-A is an 11 item interviewer rated scale that measures the severity of generalised or persistent anxiety.

Anosognosia questionnaire-dementia $(A Q-D)^{\prime}$

This instrument consists of 30 questions divided into two sections. The first section assesses intellectual functioning (for example, Do you have problems remembering dates? Do you have problems remembering telephone calls? etc), and the second section examines changes in interests and personality (for example, Do you get easily irritated? Have you lost interest in things?) Each answer is rated as never, 0 points; sometimes, 1 point; usually, 2 points; and always present, 3 points. Thus higher scores indicate more severe impairments. Form $\mathrm{A}$ is answered by the patient alone, and form $\mathrm{B}$, a similar questionnaire written in the third person, is answered by the patient's care giver blind to the patient's answers in form A. The final score is the sub- traction between scores in forms $\mathrm{B}$ and $\mathrm{A}$. Thus positive scores indicate that the caretaker rated the patient as more impaired than the patient's own evaluation (the patient was less aware of his or her cognitive or emotional deficits). We have demonstrated the reliability and validity of this scale in Alzheimer's disease. ${ }^{1}$

Bech mania scale ${ }^{15}$

This scale assesses the severity of disinhibition symptoms.

\section{Pathological laughing and crying scale (PLACS) ${ }^{16}$}

This instrument is an interviewer rated scale that quantifies aspects of pathological affect, including the duration of the episodes, their relation to external events, degree of voluntary control, inappropriateness in relation to emotions, and degree of resultant distress. Both the reliability and validity of this scale have been previously demonstrated. The scale is administered to the patient and at least one first degree relative or care giver in close contact with the patient. The scale consists of 16 items, eight assessing pathological laughter (PLACS-L) and eight assessing pathological crying (PLACS-C), which are scored from 0 to 3 points.

\section{Apathy scale ${ }^{17}$}

This scale includes 14 items which are scored by the patient's relative or care giver. Each question has four possible answers, which are scored from 0 to 3 . Thus the apathy scale score ranges from 0 to 42 points; and higher scores indicate more severe apathy. We have demonstrated the reliability and validity of the apathy scale in Alzheimer's disease. ${ }^{18}$

\section{Irritability scale s $^{18}$}

This is a 14 item scale which is rated by the patient's relative or a care giver. Scores range from 0 to 42 , and higher scores indicate more severe irritability. We have demonstrated the validity and reliability of this scale in Alzheimer's disease. ${ }^{18}$

Dementia psychosis scale (DPS) ${ }^{19}$

This is an 18 item scale which quantifies the severity and types of delusions in demented patients at the time of the psychiatric evaluation. This scale was rated by a psychiatrist with the patient and at least one close relative or care giver

\section{Functional independence measure (FIM) ${ }^{20}$}

This instrument assesses self care, sphincter control, mobility, locomotion, communication, and social cognition on a low level scale. Higher scores indicate less impairments in activities of daily living (ADLs).

\section{NEUROPSYCHOLOGICAL EXAMINATION}

The neuropsychological evaluation was carried out by a neuropsychologist who was blind to the psychiatric data. The neuropsychological battery included tests that measured deficits in different cognitive domains, such as 
verbal and visual memory, language, abstract reasoning, auditory attention, praxis, set alternation, and planning.

\section{Mini mental state examination (MMSE) ${ }^{21}$}

The MMSE is an 11 item examination that has been found to be reliable and valid in assessing a limited range of cognitive functions.

Raven's progressive matrices (RPM) 22

The RPM assesses reasoning in the visual modality. Patients are presented with a pattern problem with one part removed and several pictured inserts, one of which contains the correct pattern. The patient has to select the missing piece to complete the pattern.

\section{Wisconsin card sorting test ${ }^{23}$}

This test measures the ability to develop new concepts and shift sets, and also requires the subject to suppress a previously correct response and produce a new one. Assessment of the overall proficiency of the test was judged by the number of categories achieved (maximum $=6$ ).

\section{Controlled oral word association test ${ }^{24}$}

This test examines access to semantic information with time constraint. Patients were instructed to name as many words beginning with the letter $F$ as they could in one minute. People's names and proper nouns were not permitted. The letters $A$ and $S$ were then presented successively, one minute being allowed for each letter. The score was the number of words produced in one minute.

\section{Digit span ${ }^{25}$}

This test examines auditory attention, and consists of two parts. Both consist of seven pairs of random sequences that the examiner presents at the rate of one per second. In the first part (digits forward) the patient is asked to repeat a string of numbers exactly as it is given, and in the second (digits backwards) the patient is asked to repeat a string of numbers in reversed order.

Buschke selective reminding test ${ }^{26}$

This test measures verbal learning and memory during a multiple trial list learning task. The patient listens to a list of words and has to recall as many words as possible. Each subse-

Anosognosia questionnaire-dementia: factor loadings (varimax normalised)

\begin{tabular}{lcl}
\hline Item & Factor 1 & Factor 2 \\
\hline Recall of dates & $\mathbf{0 . 7 5}$ & $0 \cdot 01$ \\
Orientation in new places & $\mathbf{0 \cdot 7 2}$ & $0 \cdot 09$ \\
Recall of telephone calls & $\mathbf{0 \cdot 6 2}$ & $0 \cdot 08$ \\
Recall of location of objects & $\mathbf{0 . 6 7}$ & $0 \cdot 18$ \\
Handling money & $\mathbf{0 . 6 9}$ & $0 \cdot 18$ \\
Recall of appointments & $\mathbf{0 . 6 8}$ & $0 \cdot 16$ \\
Practice of hobbies & $\mathbf{0 . 6 1}$ & $0 \cdot 12$ \\
Mental calculations & $\mathbf{0 . 6 9}$ & $0 \cdot 24$ \\
Recall of shopping lists & $\mathbf{0 . 7 0}$ & $0 \cdot 06$ \\
Understanding a movie & $\mathbf{0 . 6 2}$ & $0 \cdot 27$ \\
Performance of household chores & $\mathbf{0 . 6 6}$ & $0 \cdot 17$ \\
Performance of clerical work & $\mathbf{0 . 6 5}$ & $0 \cdot 23$ \\
Increased selfishness & $0 \cdot 19$ & $\mathbf{0 . 6 1}$ \\
Increased irritability & $0 \cdot 06$ & $\mathbf{0 \cdot 7 1}$ \\
Laughing inappropriately & $0 \cdot 12$ & $\mathbf{0 . 6 0}$ \\
Increased sexual interest & $\mathbf{0 . 2 2}$ & $\mathbf{0 . 6 0}$ \\
\hline
\end{tabular}

quent learning trial involves the selective preservation of only those words that were not recalled on the immediately preceding trial. The final score was the delayed recall.

Token test ${ }^{27}$

This test examines verbal comprehension of sentences of increasing complexity.

\section{Block design ${ }^{25}$}

This test examines the presence of constructional apraxia. Patients are presented with red and white blocks and are asked to construct replicas of printed designs.

\section{Similarities ${ }^{25}$}

This test provides a measure of abstract reasoning.

\section{STATISTICAL ANALYSIS}

Statistical analysis was carried out using means and SDs, multivariate analysis of variance (ANOVA), and post hoc planned comparisons. Frequency distributions were compared using $\chi^{2}$, Fisher tests, and contingency tables. A principal components factor analysis for the AQ-D items was carried out using varimax rotation. All $\mathrm{P}$ values are two tailed.

\section{Results}

Due to lack of a care giver or relative who could complete the AQ-D, 16 patients had to be excluded from the study. Thus our sample consisted of 170 patients ( 114 women and 56 men) with a mean age (SD) of $70.5(5.4)$ years, a mean duration of illness (SD) of 3.6 (2.3) years, a mean MMSE score (SD) of $18 \cdot 8$ (6.6) points, and a mean (SD) AQ-D score of $15 \cdot 4(21 \cdot 0)$ points.

The AQ-D items of the 170 patients were entered into a principal factor analysis and a two factor solution was derived. Factor 1 had an eigenvalue of 9.34 and accounted for $31 \cdot 1 \%$ of the variance. This factor heavily loaded on the following items $(>0.60)$ : difficulties remembering dates, orienting to new places, remembering telephone calls, remembering location of objects in the house, handling money, remembering appointments, doing hobbies, performing mental calculations, remembering a shopping list, understanding the plot of a movie, carrying out household chores, and carrying out clerical work (table). Factor 2 had an eigenvalue of $2 \cdot 15$ and accounted for $7 \cdot 1 \%$ of the variance. This factor loaded on the following items $(>0.60)$ : increased selfishness, increased irritability, laughing inappropriately, and increased interest in sexual subjects (table). Thus whereas factor 1 may be construed as an unawareness of cognitive deficit factors including memory, temporal and spatial orientation, calculation, abstract reasoning, and praxis, factor 2 may be construed as an unawareness of behavioural problem factors including selfishness, irritability, inappropriateness of emotional display, and instinctive disinhibition.

One important question is whether patients 
with Alzheimer's disease show more unawareness of behavioural problems early in the disease, whereas unawareness of cognitive deficits is more prevalent in the late stages of the illness. To consider this issue we calculated a median split of the factor scores and divided patients into groups with high or low scores for each factor. Patients with high scores on factor 1 (unawareness of cognitive deficits) had a significantly longer duration of illness than patients with low scores (mean years (SD) $4 \cdot 1$ $(1.9) v 3.0(2.6)$ respectively, $F(1,168)=$ $9.51, \mathrm{p}<0.01)$, whereas no significant differences in duration of illness was found between patients with high versus low scores on factor 2 (unawareness of behavioural problems) (mean years (SD) $3.7(2.6)$ v $3.5(2.0)$ respectively, $F(1,168)=0 \cdot 23$, NS).

\section{PSYCHIATRIC CORRELATES OF ANOSOGNOSIA}

A stepwise regression analysis was calculated using factor scores from the two unawareness factors as dependent variables and age, MMSE, HAM-D, HAM-A, Bech mania scale, psychosis scale-dementia, irritability, apathy, and pathological laughing and crying scale scores as independent variables (due to missing scores on some of the above scales, 19 patients had to be excluded from the regression analyses).

Factor 1 (unawareness of cognitive deficits) showed a significant overall correlation with the independent variables $\left(\mathrm{R}^{2}=0 \cdot 26, F(10\right.$, $147)=4.99, P<0.0001)$, and the variables that accounted for a significant part of the variance were the MMSE $(\beta=-0 \cdot 18, F=$ $12.8, \mathrm{P}<0.001)$, the psychosis dementia scale $(\beta=0.21, F=8.67, P<0.01)$, the apathy scale $(\beta=0.23, F=6.17, P=0.01)$, and the HAM-D $(\beta=-0.17, F=6.73, P=$ $0 \cdot 01$ ). Factor 2 (unawareness of behavioural problems) also showed a significant overall correlation with the independent variables $\left(\mathbf{R}^{2}\right.$ $=0.17 ; F=(6,147)=5.10, P<0.0001)$. The variables that accounted for a significant part of the variance were the Bech mania scale $(\beta=0.23, F=16.0, P<0.0001)$, and the PLACS-L $(\beta=0.15, \quad F=4.83, \quad \mathrm{P}<0.05)$ (pathological laughing was present in 16 patients).

\section{NEUROPSYCHOLOGICAL CORRELATES OF} ANOSOGNOSIA

Twenty eight of the 170 patients had to be excluded from the statistical analysis due to missing data. A stepwise regression analysis was calculated, using scores of each anosognosia factor as the dependent variable and the neuropsychological tasks as independent variables. Factor 1 (unawareness-cognition) showed a significant overall correlation with the neuropsychological variables $\left(R^{2}=0.43\right.$, $F(4,137)=7.55, \mathrm{P}<0.0001)$, and the variables that accounted for a significant part of the variance were the token test $(\beta=-0 \cdot 25$, $P<0.0001)$ and the Buschke delayed recall test $(\beta=-0.24, P<0.05)$. On the other hand, there was no significant overall correlation between factor 2 (unawareness-behaviour) and the neuropsychological variables.

\section{Discussion}

We examined the presence of different domains of anosognosia in a large sample of patients with Alzheimer's disease using the anosognosia questionnaire-dementia, and there were several important findings. We found two domains of anosognosia: unawareness of cognitive deficits and unawareness of behavioural problems. Each anosognosic domain showed specific clinical correlates: whereas unawareness of cognitive deficits correlated significantly with more severe intellectual decline, a higher frequency of delusions, more severe apathy, and less depression, unawareness of behavioural problems correlated significantly with higher disinhibition scores and more severe pathological laughing. Finally, whereas unawareness of cognitive deficits correlated significantly with language and memory deficits, no significant correlations were found between unawareness of behavioural problems and neuropsychological test scores.

The first important finding of our study was the presence of two dimensions of anosognosia in Alzheimer's disease: unawareness of cognitive impairments and unawareness of behavioural problems. The question that now arises is whether these two types of unawareness have different mechanisms.

Several studies have shown significant neuropsychological correlates of anosognosia in Alzheimer's disease. Lopez et $a l^{7}$ and Michon et al ${ }^{8}$ examined patients with Alzheimer's disease with and without anosognosia using a comprehensive neuropsychological battery and found that patients with Alzheimer's disease with anosognosia had significantly more severe deficits than patients with Alzheimer's disease without anosognosia on tasks tapping frontal lobe functions. Although López et al ${ }^{7}$ and Michon et al ${ }^{8}$ suggested that anosognosia in Alzheimer's disease is related to frontal lobe dysfunction, several limitations of those studies should be pointed out. The anosognosia questionnaire used by Michon et $a l^{8}$ asked patients with Alzheimer's disease to rate the presence of cognitive abilities at the time of the evaluation compared with how they had been five years before. Thus patients were assessed about both their present awareness of cognitive deficits as well as their recall of their own past cognitive abilities. Therefore, high anosognosia scores may have been related to either memory deficits, unawareness about the current cognitive deficits, or both. Another limitation was that Michon et $a l^{8}$ only assessed anosognosia for memory deficits, whereas anosognosia for other cognitive abilities or behavioural problems was not examined. In the study of López et $a l^{7}{ }^{7}$ anosognosia was diagnosed based on the patients' answers to a single question about their cognitive status, and a structured interview of relatives or care givers was not carried out.

In the present study, we examined a large sample of patients with Alzheimer's disease using an anosognosia questionnaire with established reliability and validity. To avoid the influence of memory problems on anosog- 
nosia ratings, we asked our patients to rate their cognitive and behavioural problems as they were at the time of the evaluation. We could not find a significant correlation between domains of anosognosia and frontal lobe related tasks such as the Wisconsin card sorting test or a verbal fluency task. In a recent study, Dalla Barba et al $^{28}$ were also unable to find a significant association between deficits on frontal lobe related tasks and anosognosia in Alzheimer's disease. However, we did find a significant association between unawareness of cognitive impairments and both the token test and the Buschke selective reminding test, as well as a longer duration of illness, suggesting that deficits of verbal memory and verbal comprehension may play an important part in the production of this type of unawareness. On the other hand, there were no significant correlations between unawareness for behavioural problems and cognitive impairments, showing that neuropsychological deficits may only account for some aspects of the anosognosia syndrome in Alzheimer's disease.

Another important finding was that both types of unawareness were significantly associated with specific behavioural problems. Whereas unawareness of cognitive deficits was significantly correlated with more severe delusions and apathy and less depression, unawareness of behavioural problems was significantly correlated with more severe disinhibition and pathological laughing. In the past, anosognosia in Alzheimer's disease has been variably construed as the product of a defence mechanism protecting patients with Alzheimer's disease from depressive symptoms, ${ }^{2}$ or as a behavioural manifestation of a specific brain dysfunction. ${ }^{1}$ The first theory was based on significant, albeit mild, inverse correlations between anosognosia and depression scores-that is, less anosognosia with higher depression scores-but other studies could not replicate this correlation. ${ }^{1}$ In a recent study we found that patients with Alzheimer's disease with dysthymia had significantly lower anosognosia scores than nondepressed patients with Alzheimer's disease suggesting a greater association between relatively preserved awareness and more depression. ${ }^{1}$ However, we also found that patients with Alzheimer's disease with major depression had similar anosognosia scores that nondepressed patients with Alzheimer's disease, suggesting that some depressions in Alzheimer's disease are not related to the degree of awareness. In the present study we found a significant correlation between unawareness of cognitive deficits and depression scores-that is, higher anosognosia with lower depression scores-suggesting a role for depression in this type of anosognosia. However, depression did not account for a significant part of the variance in the correlation with unawareness of behavioural problems, suggesting that anosognosia and depression are not causally related.

Apathetic patients may be less reactive to their surroundings as well as to their needs and emotions, ${ }^{29}$ and this decreased reactivity may play a part in the production of anosognosia in Alzheimer's disease. Delusions are false beliefs that are held despite contextual evidence to the contrary, and constitute a frequent finding among patients with Alzheimer's disease. ${ }^{30}$ Delusions in Alzheimer's disease may result from dysfunction of polymodal association areas involved in the assessment of the patient's context, ${ }^{30}$ and anosognosia may occur with further disruption of brain areas related to self assessment.

We also found that unawareness of behavioural problems was significantly correlated with higher disinhibition scores and more severe pathological laughing. Thus unawareness of behavioural problems may be part of a disinhibition syndrome characterised by the release of inappropriate behaviours such as grandiose ideas, irritability, hyperactivity, and inappropriate emotional display. It is possible that the brain mechanism that controls the inhibition of behaviours may at the same time activate brain areas that mediate awareness about released or inhibited behaviours. Disruption of this system may result in a disinhibition syndrome and anosognosia for behavioural problems.

There are clinical implications of our findings. Johnson and Orrell ${ }^{31}$ suggested that patients' perceptions about their illness may be influenced by their own perception of the disease, the treatment they receive, and the quality of psychiatric services and social supports available to them. These important issues should be considered in future studies. In a recent study that assessed insight in schizophrenic patients, David et $a^{32}$ suggested an indirect approach to help patients recognise their illness. Future studies should assess the utility of these techniques in patients with Alzheimer's disease, as these interventions may improve their quality of life and lessen the care givers' burden.

In conclusion, we identified two domains of anosognosia in Alzheimer's disease: unawareness of cognitive deficits and unawareness of behavioural problems, and we demonstrated different cognitive and psychiatric correlates for these disorders. Future studies should examine neuroradiological and neurometabolic correlates of these anosognosic domains in Alzheimer's disease.

This study was partially supported by a grant from the Raúl Carrea Institute of Neurological Research and the Fundacion Perez Companc. We thank Dr J Paul Fedoroff for his most helpful comments.

1 Migliorelli R, Teson A, Sabe L, et al. Anosognosia in Alzheimer's disease: a study of associated factors. $\mathcal{f}$ Neuropsychiatry Clin Neurosci 1995;7:338-44.

2 Reisberg B, Gordon B, McCarthy M. Insight and denial accompanying progressive cognitive decline in normal aging and Alzheimer's disease. In: Stanley B, ed. Geriatric psychiatry: ethical and legal issues. Washington, DC: American Psychiatric Press, 1985:19-39

3 Feher EP, Mahurin RK, Inbody SB, Crook TH, Pirozzolo F. Anosognosia in Alzheimer's disease. Neuropsychiatry, Neuropsychology and Behavioral Neurology 1991;4:136-46.

4 Sevush S, Leve N. Denial of memory deficit in Alzheimer's disease. Am ₹ Psychiatry 1993;150:748-51.

5 Reed BR, Jagust WJ, Coulter L. Anosognosia in Alzheimer's disease: relationships to depression, cognitive function and corbral Neuropsychol, and cerebral

6 Vasterling JF, Seltzer B, Foss JF, Vanderbrook V. Unawareness of deficit in Alzheimer's disease: domainspecific differences and disease correlates. Neuro- 
psychiatry, Neuropsychology, and Behavioral Neurology 1995;8:26-32.

7 López OL, Becker JT, Somsak D, Dew MA, DeKosky ST. Awareness of cognitive deficits in probable Alzheimer's disease. Eur Neurol 1993;34:277-82.

8 Michon A, Deweer B, Pillon B, Agid Y, Dubois B. Relation of anosognosia to frontal lobe dysfunction in Alzheimer's disease. $\mathscr{f}$ Neurol Neurosurg Psychiatry 1994;57:805-9.

9 McKhann G, Drachman D, Folstein MF, Katzman R, Price D, Stadlan EM. Clinical diagnosis of Alzheimer's disease: report of the NINCDS-ADRDA Work Group under the auspices of Department of Health and Human Services Task Force on Alzheimer's Disease. Neurology 1984;34:939-44.

10 Hachinski VC, Iliff LD, Zilhka L, et el. Cerebral blood flow in dementia. Arch Neurol 1975;40:97-103.

11 Spitzer RL, Williams JBW, Gibbon M, First MB. The structured clinical interview for DSM-III-R SCID. I: hisstructured clinical interview for DSM-III-R SCID. I: history, rationale,

12 American Psychiatric Press. Diagnostic and statistical manual of mental disorders. 3rd ed, revised. Washington, DC: APA, 1987.

13 Hamilton MA. A rating scale for depression. $f$ Neurol Neurosurg Psychiatry 1960;23:56-62.

14 Hamilton MA. The assessment of anxiety states by rating. Br f Med Psychol 1959;32:50-5.

15 Bech $P$, Kastrup M, Rafaelsen OJ. Mini-compendium of rating scales for states of anxiety, depression, mania, schizophrenia, with corresponding DSM-III syndromes. Acta Psychiatr Scand 1986;73(suppl 236):29-31.

16 Robinson RG, Parikh RM, Lipsey JR, Starkstein SE, Price TR. Pathological laughing and crying following stroke:
validation of measurement scale and double blind treatvalidation of measurement scale and double blin
ment study. Am $\mathcal{f}$ Psychiatry 1993;150:286-93.

17 Starkstein SE, Mayberg HS, Preziosi TJ, Andrezejewski MA, Leiguarda R, Robinson RG. Reliability, validity, and clinical correlates of apathy in Parkinson's disease. $\mathcal{f}$ Neuropsychiatry Clin Neurosci 1992;4:134-9.

18 Starkstein SE, Migliorelli R, Manes $F$, et al. The prevalence and clinical correlates of apathy and irritability in Alzheimer's disease. Fournal of European Neurology 1995 2:540-6.

19 Migliorelli R, Petracca G, Teson A, Sabe L, Leiguarda R Starkstein SE. Neuropsychiatric and neuropsychologica correlates of delusions in Alzheimer's disease. Psycho Med 1995;25:505-13.

20 Granger CV, Hamilton BB, Kayton R. Guide for use of the uniform data set for medical rehabilitation. Uniform data system for medical rehabilitation. Buffalo: 1986.

21 Folstein MF, Folstein SE, McHugh PR. Mini-mental state: a practical method for grading the cognitive state of
patients for the clinician. $f$ Psychiatr Res $1975 ; 12: 189-98$.

22 Raven JC, Court JH, Raven J. Manual for Raven's progressive matrices and vocabulary scales. London: HK Lewis, 986

23 Nelson HE. A modified card sorting test sensitive to frontal lobe defects. Cortex 1976;12:313-24.

24 Benton AL. Differential behavioral effects in frontal lobe disease. Neuropsychology 1968;6:53-60.

25 Wechsler D. Wechsler adult intelligence scale manual. New York: Psychological Corporation, 1955.

26 Buschke H, Fuld PA. Evaluating storage, retention, and retrieval in disordered memory and learning. Neurolog 1974;24:1019-25.

27 De Renzi E, Faglioni P. Development of a shortened version of the token test. Cortex 1978;14:41-9.

28 Dalla Barba G, Parlato V, Iavarone A, Boller F. Anosognosia, intrusions and 'frontal' functions in Alzheimer's disease and depression. Neuropsychology 1995;33:247-59.

29 Marin RS. Apathy: a neuropsychiatric syndrome. Neuropsychiatry Clin Neurosci 1991;3:243-54.

30 Starkstein SE, Vazquez S, Petracca G, Teson A, Migliorelli R, Leiguarda R. A SPECT study of delusions in R, Leiguarda R. A SPECT study of delu
Alzheimer's disease. Neurology 1994;44:2055-9.

31 Johnson S, Orrell M. Insight: a social perspective. Psychol Med 1995;25:515-20.

32 David A, Buchanan A, Ried A, Almeida O. The assessmen of insight in psychosis. $B r f$ Psychiatry 1992;161: 599-602 